ISSN 1112-9867

http://www.jfas.info

\title{
SYNTHESIS OF MCM-41 NANOMATERIAL FROM ALGERIAN BENTONITE: INFLUENCE OF SYNTHESIS pH
}

\author{
T. Ali-Dahmane ${ }^{1,2, *}$, L. Brahmi ${ }^{3,4}$, R. Hamacha ${ }^{1}$, F. Villieras ${ }^{5}$ and A. Bengueddach ${ }^{1}$ \\ ${ }^{1}$ Laboratoire de Chimie des Matériaux, Université d'Oran 1 Ahmed Ben Bella, Oran, Algérié. \\ ${ }^{2}$ École Supérieure en Sciences Appliquées de Tlemcen, Tlemcen, Algérie. \\ ${ }^{3}$ Laboratoire de Chimie Fine, Université d'Oran 1 Ahmed Ben Bella, Oran, Algérie. \\ ${ }^{4}$ Université A Belkaid, Tlemcen, Algérie. \\ ${ }^{5}$ Laboratoire Environnement et Minéralurgie, François-Fiessinger Research, Nancy, France.
}

Received: 10 December 2016 / Accepted: 08 April 2017 / Published online: 01 May 2017

\begin{abstract}
Mesoporous materials of the MCM-41 type were synthesized from Algerian bentonite as an aluminosilicate source without the addition of pure silica and aluminum reagents. The samples were synthesized under hydrothermal condition using cetyltrimithylammonium bromide (CTAB) as surfactant. The influence of initial synthesis $\mathrm{pH}$ on the material properties of as-synthesis MCM-41 samples was studied at different temperatures of crystallization. The samples were characterized using several techniques e.g. X-ray diffraction (XRD), $\mathrm{N}_{2}$ adsorption-desorption and transmission electron microscope (TEM). The experimental results showed that bentonite can be used as an aluminosilicate source for the synthesis of Al-MCM-41. By decreasing the synthesis temperature of crystallization, it is necessary to decrease the synthesis $\mathrm{pH}$ value to form the best ordered hexagonal phase and eliminate the lamellar phase.
\end{abstract}

Keywords: bentonite; alkaline fusion; pH; Al-MCM-41.

Author Correspondence, e-mail: byalidahmane@gmail.com

doi: http://dx.doi.org/10.4314/jfas. v9i2.2 


\section{INTRODUCTION}

Pour la synthèse de MCM-41 on utilise généralement des organosilicates (tetramethylorthosilicate ou tetraethylorthosilicate,...), silice colloïdale ou fumée de silice comme des sources de silice [1-5]. Ces dernières années et pour des considérations économiques et environnementales, les chercheurs étudient la possibilité de remplacer ces sources de silice par des minéraux naturels largement disponibles et peu onéreux.

Singer et coll. [6] ont proposé de convertir les cendres de charbon (coal fly ash) par des méthodes d'activation hydrothermale des zéolites en utilisant des solutions alcalines (telles que $\mathrm{NaOH}$ ou $\mathrm{KOH}$ ) qui sont analogues à la formation des zéolithes naturelles à partir des dépôts volcaniques. En 1999, Chang et coll. [7] ont étudié la conversion des cendres de charbon en matériaux mésoporeux aluminosilicates de type MCM-41, avec une incorporation importante de l'aluminium $(\mathrm{Si} / \mathrm{Al}=13.4)$. Kumar et coll. [8], synthétisent MCM-41 et SBA-15 avec des surfaces spécifiques de l'ordre $842 \mathrm{~m}^{2} / \mathrm{g}$ et $483 \mathrm{~m}^{2} / \mathrm{g}$ respectivement à partir des cendres de charbon, en utilisant la méthode de fusion. Liu et coll. [9] utilisent NaY et le kaolin comme des matériaux de départ pour la synthèse d'un composite kaolin/NaY/MCM-41 qui présente une stabilité hydrothermale importante. Kang et coll. [10], réalisent la synthèse de MCM-41 en utilisant le silicate de sodium comme source de silice et metakaolin comme la seule source d'aluminium. En 2005, Miao et coll. [11], ont synthétisé un matériau mésoporeux aluminosilicaté à partir de K-feldspar comme source de silice et d'aluminium. Les parois du produit obtenu sont similaires à celle des zéolites et l'aluminium incorporé est de forme tétraédrique. Sanhueza et coll. [12], utilisent la diatomite et la pumicite pour la synthèse de MCM-41. Dans le cas de la diatomite, le $\mathrm{NaAlO}_{2}$ est utilisé pour ajuster le rapport $\mathrm{Si} / \mathrm{Al}$. Wu et coll. [13], ont utilisé la microcline comme une source de silice et d'aluminium pour la synthèse des matériaux mésoporeux. Ils obtiennent un matériau mésoporeux avec des parois de la zéolithe 13X. En 2009, une bentonite de type volclay a été utilisée comme source de silice et d'aluminium pour la préparation de Al-MCM-41 [14]. Le matériau obtenu a une surface spécifique très importante de l'ordre de $1060 \mathrm{~m}^{2} / \mathrm{g}$. Dans une autre étude, Yang et coll., [15] ont utilisé la bentonite de Chine comme une source aluminosilicatée pour la synthèse de Al-MCM-41, en adoptant la fusion alcaline pour extraire 
la silice et l'aluminium. Les résultats sont encourageants : une $S_{\mathrm{BET}}$ de l'ordre de $1018 \mathrm{~m}^{2} / \mathrm{g}$ et un rapport $\mathrm{Si} / \mathrm{Al}=36$. Une étude publiée en 2012 par I. Majchrzak - Kcueba [16], montre l'utilisation des cendres de charbon pour la synthèse de Al-MCM-41. Les surfaces spécifiques des matériaux obtenus varient entre 350 et $610 \mathrm{~m}^{2} / \mathrm{g}$ avec un rapport $\mathrm{Si} / \mathrm{Al}=13.5$. En 2013, un matériau de type MCM-41 purement silicique à été synthétisé en utilisant la chrysotile comme une source de silice [17]. Dans la même année, Ghorbani et coll. [18], ont pu préparer une MCM-41 avec une grande qualité en utilisant une plante nommée carex comme source de silice.

Dans nos travaux précedent, nous avons synthétisés Al-MCM-41 par la bentonite allemande nommée volclay [14]. En 2014, une étude comparative a été consacrée entre la bentonite algérienne et la bentonite volclay comme des sources aluminosilicatées pour la synthèse de Al-MCM-41 sans étudier aucuns paramètres de synthèse [19]. Dans cette étude, notre but est d'optimiser quelques paramètres de synthèse à savoir le $\mathrm{pH}$ initial de synthèse et la température de cristallisation pour la synthèse de Al-MCM-41 en utilisant une bentonite algérienne comme une source de silice et d'aluminium.

\section{RESULTS AND DISCUSSION}

\subsection{Analyse par diffraction RX}

Les diffractogrammes $\mathrm{RX}$ des échantillons non calcinés préparés à $80^{\circ} \mathrm{C}$ et à différents $\mathrm{pH}$ de synthèse sont illustrés dans la figure (1A). L'analyse de ces produits sur un intervalle de 2théta entre 2 et 35(20), montre qu'aucun des pics correspondant aux phases de la bentonite ne sont présents. Par conséquent, La majeure quantité extraite de silice et d'aluminium de la bentonite a été consommée pour produire Al-MCM-41. Le matériau mésoporeux de type MCM-41 est obtenu pour tous les milieux étudiés, caractérisé par un pic à des faibles angles RX qui correspond à la réflexion $(100)$ à 2 théta $=2^{\circ}[20] . \mathrm{A} \mathrm{pH}>8.5$, les échantillons présentent d'autres réflexions indexées par $(*)$ à 2théta $=10.3,20.6$ et $31.4(2 \theta)$, elles caractérisent probablement une phase lamellaire. L'espace entre les feuillets de cette phase doit être rempli par l'agent structurant, puisque la structure est complètement détruite après une calcination à $550{ }^{\circ} \mathrm{C}$. 
La figure (1B), illustre les diffractogrammes des échantillons préparé à $100{ }^{\circ} \mathrm{C}$ et à différents pH. Ces diffractogrammes montrent que MCM-41 est obtenu pour tous les milieux. L'utilisation d'une température de synthèse de $100{ }^{\circ} \mathrm{C}$, fait apparaitre la phase lamellaire pour un $\mathrm{pH}$ élevé $(\mathrm{pH}=11.5)$.

Les diffractogrammes RX (figure 1C) des échantillons préparés à température $130^{\circ} \mathrm{C}$, montrent qu'il n'y a pas d'apparition de la phase lamellaire. Cela suggère que les températures de synthèse élevées $\left(\mathrm{T}>80^{\circ} \mathrm{C}\right)$ défavorisent la formation de la phase lamellaire.
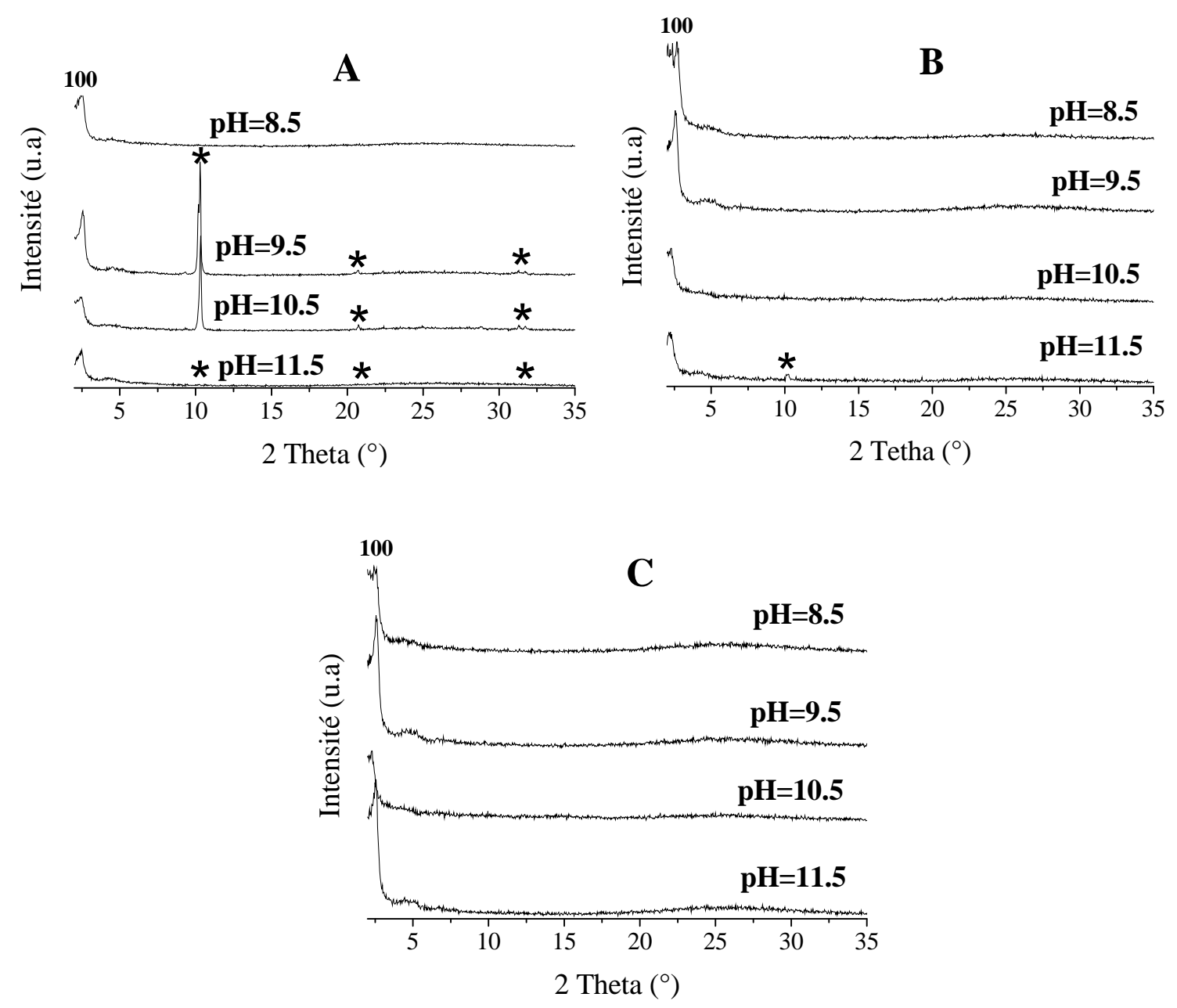

Fig.1. Diffractogrammes RX des MCM-bentonite non calcinée à différentes températures de synthèse: $\mathbf{A}\left(80^{\circ} \mathrm{C}\right), \mathbf{B}\left(100^{\circ} \mathrm{C}\right)$ et $\mathbf{C}\left(130^{\circ} \mathrm{C}\right)$.

Les concentrations élevées en ions $\mathrm{Na}^{+}$dans la solution précurseur, favorisent la formation des zéolithes et défavorisent celle des matériaux mésoporeux [21]. L'ajustement du pH durant la synthèse de MCM-Bentonite est très important suite à la concentration élevée des ions $\mathrm{Na}^{+}$ 
présents dans la solution surnageante de la bentonite fondue [22].

Luchinger et coll. [22], ont étudié l'effet positif du traitement acide sur la structure de MCM-41. Ce traitement déplace l'équilibre de polymérisation/dépolymérisation des espèces silicates à un degré de condensation élevé. Il aide à rapprocher les silicates autour des micelles en les liant par la formation de liaisons covalentes de type $\mathrm{Si}-\mathrm{O}-\mathrm{Si}$. Comme deuxième effet, la diminution du $\mathrm{pH}$ donne la protonation de la charge négative des espèces silicates pour réduire la répulsion électrostatique entre les micelles. Ceci facilite l'approche et la condensation des micelles.

Le traitement acide réduit aussi la quantité des charges qui compensent les ions $\mathrm{Na}^{+}$restant sur le squelette de MCM-41. Après protonation, les unités Si-O-Na deviennent des groupes silanol Si-OH. Les groupes silanol adjacents se condensent dans l'étape de calcination par élimination d'eau. De cette façon, l'ordre structural de la MCM-41 peut s'améliorer au cours de l'étape de calcination, si le traitement acide est appliqué de façon appropriée. Busio et coll. [23] ont étudié l'incorporation d'aluminium dans MCM-41 en utilisant des réactifs purs (CTAB, silicate de sodium et sulfate d'aluminium). Ils ont interprété l'apparition de la phase lamellaire par la forte incorporation d'aluminium $(\mathrm{Si} / \mathrm{Al}=5)$.

L'étude réalisée par K.S. Hui et coll. [24], sur la synthèse de MCM-41 à partir de coal fly ash, a montré que la quantité d'aluminium incorporé augmente avec le $\mathrm{pH}$ de synthèse. Donc on peut dire que la synthèse à la température de $80{ }^{\circ} \mathrm{C}$, favorise l'incorporation d'aluminium, mais laisse apparaitre la phase lamellaire suite à la forte incorporation d'aluminium.

En augmentant la température de synthèse à 100 ou $130{ }^{\circ} \mathrm{C}$, il n'y a pas d'apparition de la phase lamellaire, sauf l'échantillon préparé à $100{ }^{\circ} \mathrm{C}$ pour un $\mathrm{pH}$ élevé $(\mathrm{pH}=11.5)$. Cela suggère que les températures de synthèse élevées $\left(\mathrm{T}>100^{\circ} \mathrm{C}\right)$ défavorisent la formation de la phase lamellaire. En se basant sur ces résultats, les échantillons optimisés sont représentés sur la figure (2) et le tableau (1). 


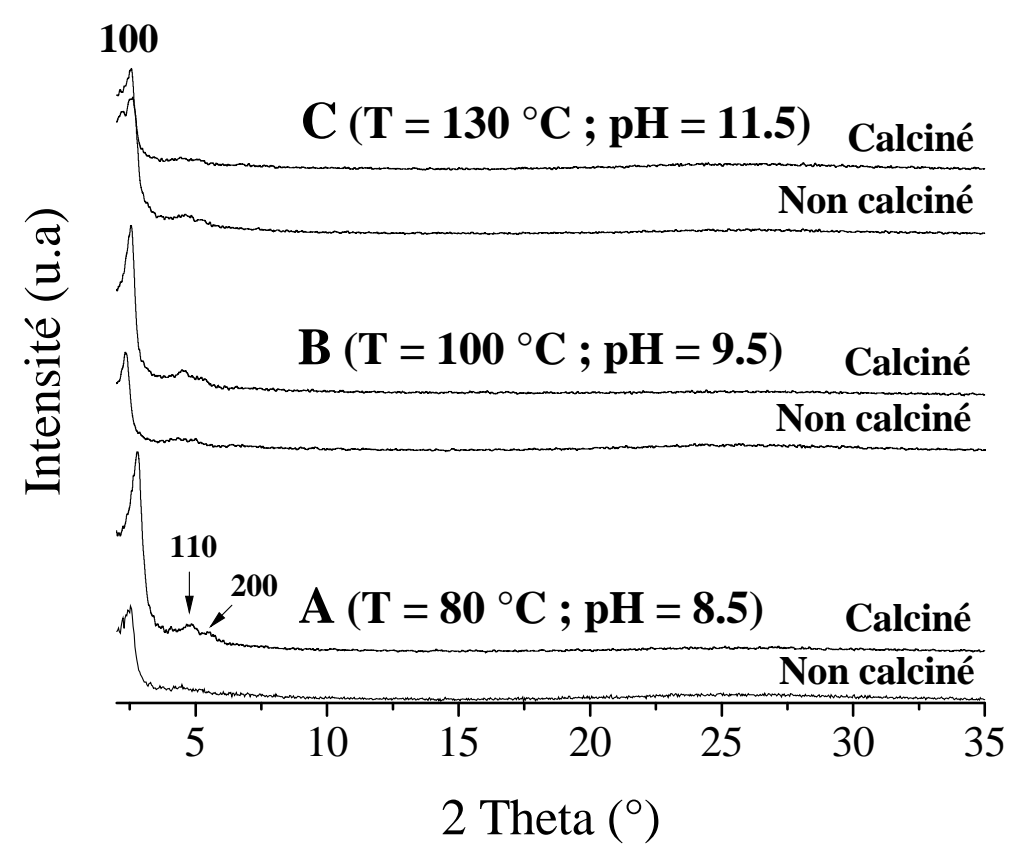

Fig.2. Diffractogrammes RX des MCM-bentonite obtenus à partir de conditions de synthèse ( $\mathrm{pH}$, température) optimisées.

Tableau 1. Données de DRX des échantillons MCM-bentonite.

\begin{tabular}{|c|c|c|c|c|c|c|c|}
\hline \multirow{2}{*}{$\begin{array}{l}\text { Température } \\
\left({ }^{\circ} \mathrm{C}\right)\end{array}$} & \multirow{2}{*}{$\begin{array}{c}\mathrm{pH} \text { de } \\
\text { synthèse }\end{array}$} & \multicolumn{3}{|c|}{ Non calciné } & \multicolumn{3}{|c|}{ Calciné } \\
\hline & & $\begin{array}{c}2 \theta \\
(100)\end{array}$ & $\begin{array}{l}\mathrm{d}_{100} \\
(\AA)\end{array}$ & $\begin{array}{c}\mathrm{a}_{0} \\
(\AA)\end{array}$ & $\begin{array}{l}\text { 2theta } \\
(100)\end{array}$ & $\begin{array}{l}\mathrm{d}_{100} \\
(\AA)\end{array}$ & $\begin{array}{c}\mathrm{a}_{0} \\
(\AA)\end{array}$ \\
\hline 80 & 8.5 & 2.42 & 42.36 & 48.92 & 2.80 & 36.61 & 42.28 \\
\hline 100 & 9.5 & 2.32 & 44.18 & 51.08 & 2.69 & 38.10 & 44.00 \\
\hline 130 & 11.5 & 2.56 & 40.04 & 46.24 & 2.63 & 38.98 & 45.02 \\
\hline
\end{tabular}

$\mathrm{d}_{100}$ : distance interréticulaire, calculé par la relation $\mathrm{n} \lambda=2 \mathrm{~d}_{100} \sin \theta$.

$\mathrm{a}_{0}$ : unité de maille, $\mathrm{a}_{0}=2 \mathrm{~d}_{100} / \sqrt{3}$.

\section{2. $\mathrm{N}_{2}$ adsorption-désorption}

La figure (3) représente les isothermes adsorption-désorption d'azote des MCM-bentonite et le tableau (2) résume les propriétés texturales de ces échantillons. Les isothermes obtenues sont de type IV caractéristique des solides mésoporeux selon la nomenclature IUPAC [25]. L'étape d'inflexion correspondant à la condensation capillaire dans les mésopores, se situe entre 0.21-0.35 en $\mathrm{P} / \mathrm{P}_{0}$ typique d'un matériau mésoporeux.

Tous les isothermes d'adsorption-désorption d'azote présentent une hystérésis après la 
condensation capillaire qui caractérise le domaine mésoporeux à des pressions relatives 0.45-1.00 en $\mathrm{P} / \mathrm{P}_{0}$. Cette hystérèse est causée par une condensation capillaire dans les méso-ou macropores qui existent entre les particules. Des résultats similaires ont été obtenus par Yang [15] et Majchrzak-Kuçeba [16].

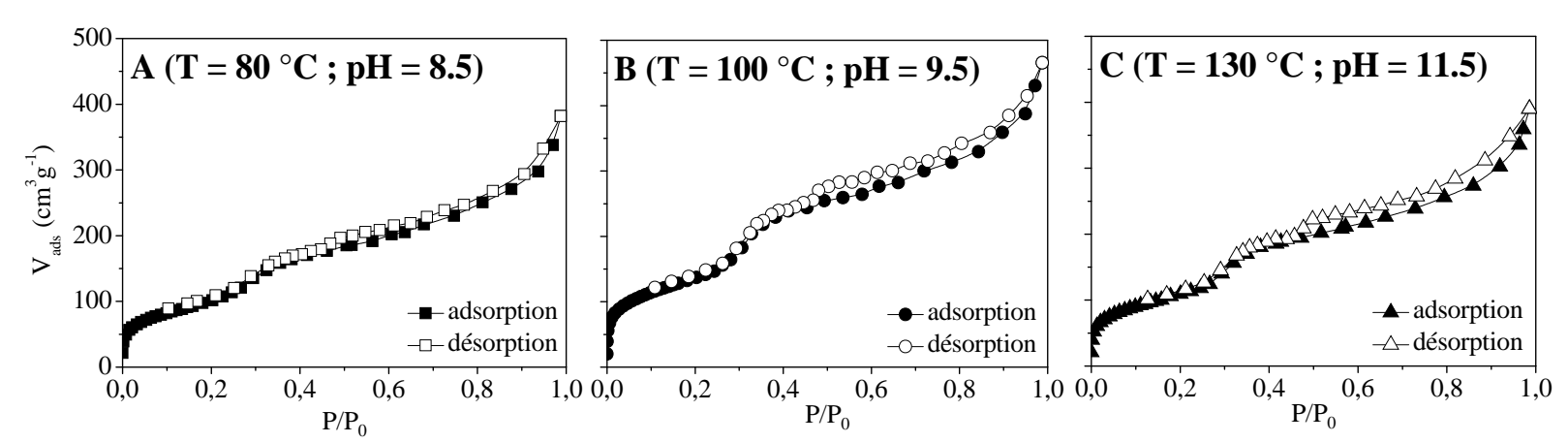

Fig.3. Isothermes adsorption-désorption de $\mathrm{N}_{2}$ des MCM-bentonite obtenus à partir de conditions de synthèse ( $\mathrm{pH}$, température) optimisées.

Pour mieux estimer le taux d'incorporation de l'aluminium dans nos matériaux, il nous a semblé nécessaire de faire la différence entre le rapport Si/Al dans la partie amorphe et celui de la partie cristalline.

Comme le montre le tableau (2), le rapport $\mathrm{Si} / \mathrm{Al}$ des matériaux mésoporeux sélectionnés préparés à partir de la bentonite est entre 8.1 (1) à 13.0 (3) pour les parties amorphes. Ces rapports sont plus élevés que le Si/Al de la bentonite naturelle (4.7) et proche de celui de la bentonite fondue (8.07). Apparemment, la température et le rapport bentonite/ $\mathrm{NaOH}$ choisis pour la fusion alcaline ne nous permettent pas d'extraire complètement l'aluminium. La synthèse d'un matériau mésoporeux sous les conditions de température $\left(80{ }^{\circ} \mathrm{C}\right)$ et $\mathrm{pH}=8.5$, nous donne l'échantillon (1) avec une incorporation d'aluminium presque totale dans la partie amorphe $(\mathrm{Si} / \mathrm{Al}=8.11)$ comparée avec celui de la bentonite fondue $(8.07)$, mais un rapport $\mathrm{Si} / \mathrm{Al}$ de 99 dans la partie cristalline.

L'incorporation élevée d'aluminium est la cause des faibles surfaces spécifiques enregistrées pour ces échantillons. D’après les données de la littérature [26], quelle que soit la source d'aluminium utilisée pour la synthèse des matériaux mésoporeux aluminosilicates de type MCM-41 à partir des réactifs purs, la surface spécifique et le diamètre des pores diminuent toujours quand la quantité d'aluminium introduite est élevée. 
Tableau 2. Caractéristiques structurales des MCM-bentonite calcinés.

\begin{tabular}{|c|c|c|c|c|c|c|c|c|}
\hline Echantillons & $(\mathrm{Si} / \mathrm{Al}) *$ & $(\mathrm{Si} / \mathrm{Al})$ & $\begin{array}{c}\mathrm{S}_{\mathrm{BET}}{ }^{\mathrm{a}} \\
\left(\mathrm{m}^{2} / \mathrm{g}\right)\end{array}$ & $\begin{array}{c}\mathrm{V}_{\mathrm{p}}^{\mathrm{b}} \\
\left(\mathrm{cm}^{3} / \mathrm{g}\right)\end{array}$ & $\begin{array}{l}\mathrm{D}_{\mathrm{p}}^{\mathrm{c}} \\
(\AA)\end{array}$ & $\begin{array}{l}\mathrm{d}_{100} \\
(\AA)\end{array}$ & $\begin{array}{c}\mathrm{a}_{\mathrm{o}} \\
(\AA)\end{array}$ & $\begin{array}{l}\mathrm{E}_{\mathrm{p}}^{\mathrm{d}} \\
(\AA) \\
\end{array}$ \\
\hline (1): $\mathrm{T}=80^{\circ} \mathrm{C}, \mathrm{pH}=8.5$ & 8.11 & 98.99 & 373 & 0.59 & 33.37 & 36.61 & 42.28 & 8.91 \\
\hline (2): $\mathrm{T}=100^{\circ} \mathrm{C}, \mathrm{pH}=9.5$ & 12.43 & 43.64 & 494 & 0.72 & 38.00 & 38.10 & 44.00 & 6.00 \\
\hline (3): $\mathrm{T}=130^{\circ} \mathrm{C}, \mathrm{pH}=11.5$ & 13.06 & 30.12 & 417 & 0.60 & 35.66 & 38.98 & 45.01 & 9.35 \\
\hline Bentonite naturelle & 4.76 & - & - & - & - & - & - & - \\
\hline Bentonite fondue & 8.07 & _ & - & - & - & - & - & - \\
\hline
\end{tabular}

(Si/Al)* : Rapport dans la partie amorphe ; $\mathbf{S i} / \mathbf{A l})$ : rapport dans la partie cristalline: déduits de l'analyse EDX.

${ }^{a}$ Surface spécifique.

${ }^{\mathrm{b}}$ Volume mésoporuex.

${ }^{c}$ Diamètres des pores déterminé par BJH.

${ }^{\mathrm{d}}$ Epaisseur des parois, $\mathrm{E}_{\mathrm{p}}=\mathrm{a}_{0}-\mathrm{Dp}$.

Il est à noter qu'en diminuant la température de synthèse, il est nécessaire de diminuer la valeur du $\mathrm{pH}$ de synthèse pour former la phase hexagonale la mieux ordonnée et éliminer la phase lamellaire.

L'apparition des pics qui correspondent à la réflexion (110) et (200), témoignent d'une meilleure structuration hexagonale des canaux d'Al-MCM-41. Après calcination, la position de 2 théta est déplacée à des valeurs élevées pour tous les échantillons suite à la contraction de l'unité de maille (tableau 2). Ce phénomène est causé par le départ de l'agent structurant, et par la condensation des groupes silanols des parois [22, 27, 28].

\subsection{Microscope électronique à transmission}

La figure (4), montre des photos obtenues par microscopie électronique à transmission de la bentonite fondue et MCM-bentonite. Les images confirment que le matériau mésoporeux présente un système de pores uniforme et une distribution hexagonale très claire. 

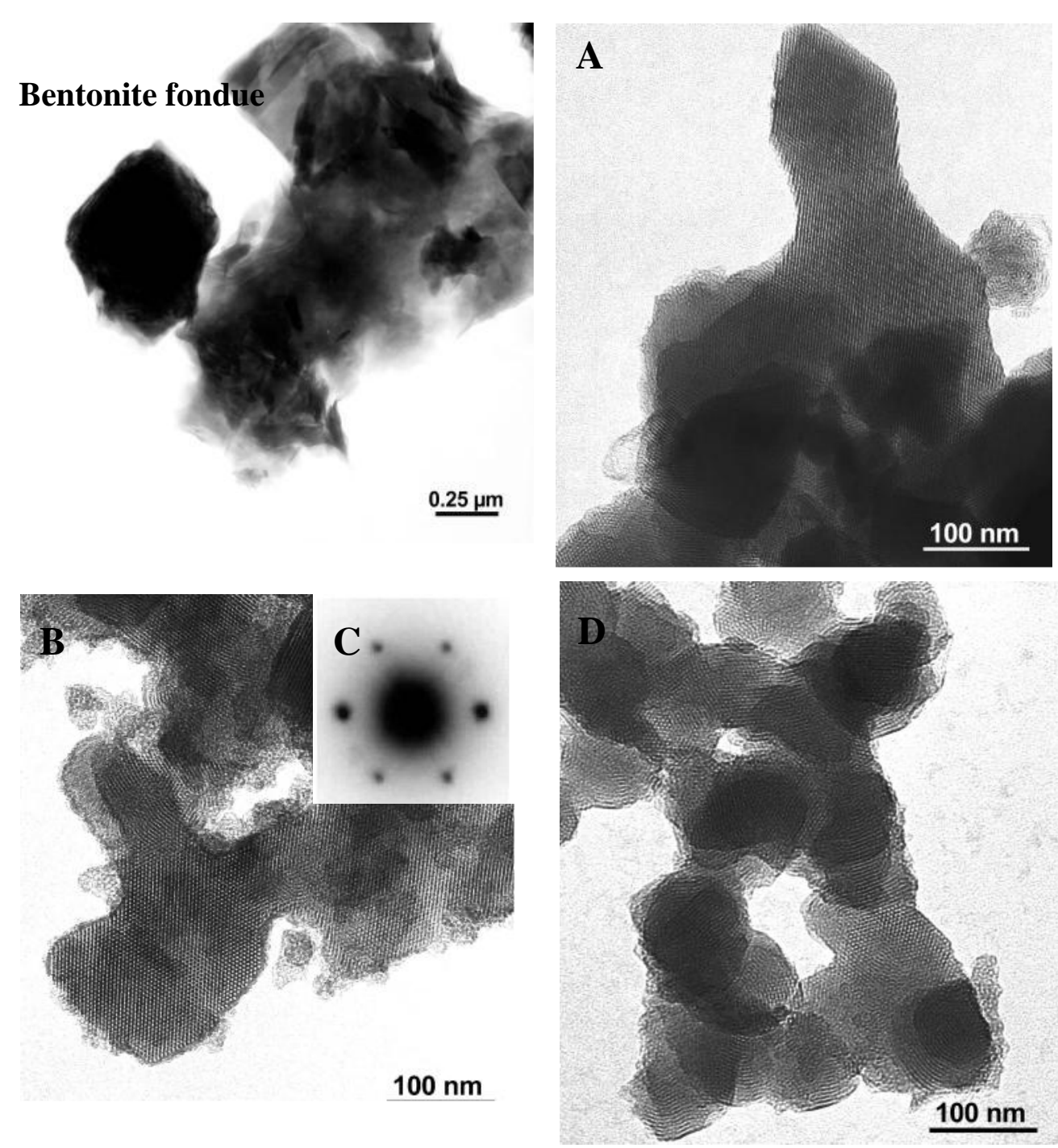

Fig.4. Images obtenues par MET des MCM-bentonite calcinés: $\mathbf{A}\left(\mathrm{T}=80{ }^{\circ} \mathrm{C} ; \mathrm{pH}=8.5\right), \mathbf{B}(\mathrm{T}$ $\left.=100{ }^{\circ} \mathrm{C} ; \mathrm{pH}=9.5\right), \mathbf{C}($ Diffraction électronique $)$ et $\mathbf{D}\left(\mathrm{T}=130^{\circ} \mathrm{C} ; \mathrm{pH}=11.5\right)$.

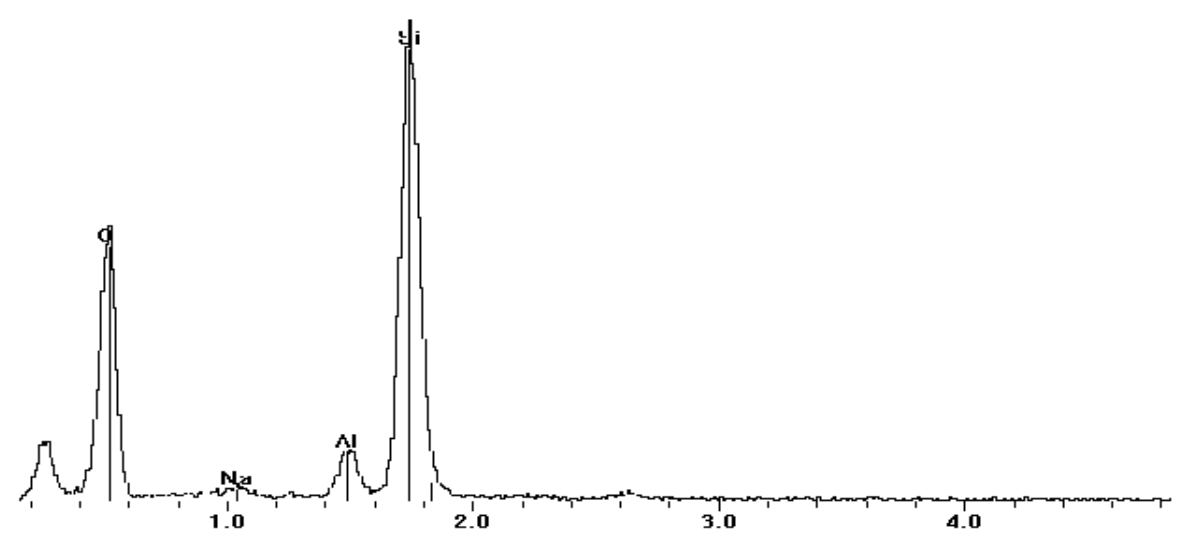

Fig.5. Spectre EDX dans la partie cristalline de MCM-bentonite $\left(\mathrm{T}=100{ }^{\circ} \mathrm{C}\right.$ et $\left.\mathrm{pH}=9.5\right)$. 
Ce résultat est bien corrélé avec la DRX, et par la diffraction électronique de l'échantillon préparé à $100{ }^{\circ} \mathrm{C}$ et $\mathrm{pH}=9.5$ (figure $4 \mathrm{C}$ ). La valeur de l'unité de maille égale à $42 \AA$ est très bien corrélée à celle obtenue par diffraction des rayons X (44 Å). Par analyse EDX (figure 5), nous confirmons qu'aucune impureté n'est détectée telle que $\mathrm{Fe}, \mathrm{Ca}$, ou $\mathrm{K}$, donc le matériau obtenu est purement aluminosilicaté.

\section{EXPERIMENTAL}

\subsection{Origine et la composition chimique de la bentonite Algérienne}

La bentonite naturelle utilisée dans ce travail, a été ramenée du gisement de HAMMAM BOUGHRARA, qui est située à $15 \mathrm{~km}$ au Nord-Est de la ville de MAGHNIA et à $3 \mathrm{~km}$ au Nord-Ouest du village de HAMMAM BOUGHRARA. Sa composition chimique est donnée sur le tableau (3).

Tableau 3. Composition chimique de la bentonite naturelle.

\begin{tabular}{lcccccccc}
\hline Composition (\% massique) & $\mathrm{SiO}_{2}$ & $\mathrm{Al}_{2} \mathrm{O}_{3}$ & $\mathrm{Fe}_{2} \mathrm{O}_{3}$ & $\mathrm{MgO}$ & $\mathrm{TiO}_{2}$ & $\mathrm{CaO}$ & $\mathrm{Na}_{2} \mathrm{O}$ & $\mathrm{K}_{2} \mathrm{O}$ \\
\hline Bentonite naturelle & 60.49 & 18.59 & 2.29 & 3.80 & 0.10 & 1.00 & 1.20 & 0.90 \\
\hline
\end{tabular}

Les composants majeurs de la bentonite sont la silice et l'aluminium, avec des quantités faibles d'autres éléments tels que $\mathrm{Fe}, \mathrm{Mn}, \mathrm{Ca}, \mathrm{Na}, \ldots \mathrm{La}$ silice $\left(\mathrm{SiO}_{2}\right)$ dans la bentonite est majoritaire $60.5 \%$, suivie par l'alumine $\left(\mathrm{Al}_{2} \mathrm{O}_{3}\right)$ qui est de l'ordre de $18.6 \%$.

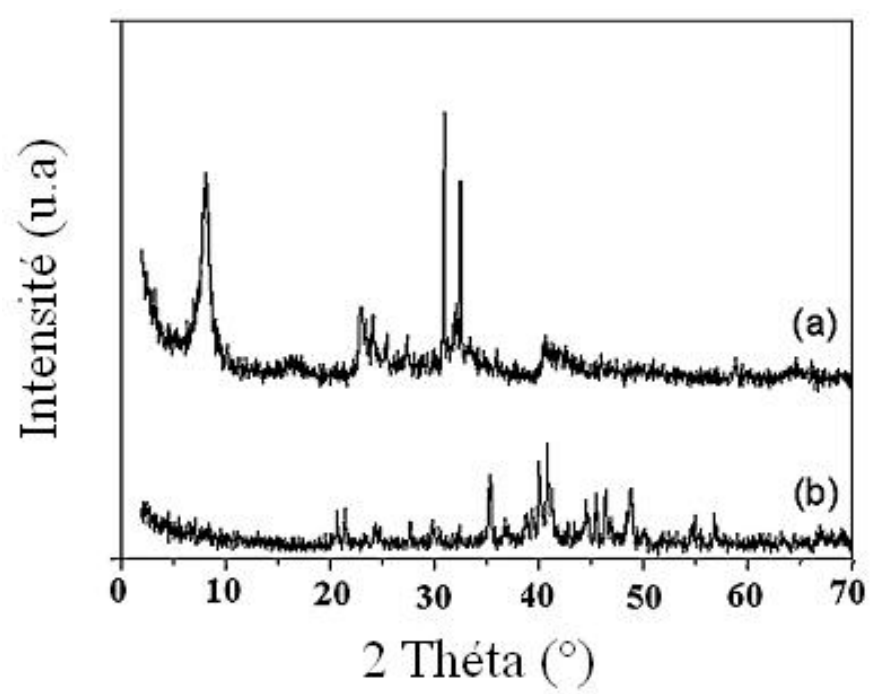

Fig.6. Diffractogramme RX de la bentonite (a) et la bentonite fondue à $550^{\circ} \mathrm{C}$ (b). 
Par diffraction des RX (figure 6a), on remarque que deux minéraux argileux, la montmorillonite et la kaolinite sont prédominants dans la bentonite naturelle de même qu'on relève la présence du quartz un minéral non argileux (tableau 4). Dans la bentonite fondue à $550^{\circ} \mathrm{C}$, les constituants minéralogiques majeurs de la bentonite brute ((Mont), (K) et (Q) disparaissent après fusion (figure $6 b$ ).

Tableau 4. Phases minérales dans la bentonite.

\begin{tabular}{c|lrc|c}
\hline Nature & \multicolumn{3}{|c|}{ Minéraux argileux } & Minéraux non argileux \\
\hline Bentonite & Mont & \multicolumn{1}{c}{ I } & K & Q \\
\hline \multirow{3}{*}{$\mathrm{d}(\AA)$} & 12.65 & 10 & 7.16 & 4.25 \\
& 4.43 & 5 & 3.57 & 3.34 \\
& 2.95 & - & 2.37 & 2.45 \\
& 2.52 & - & - & 2.28 \\
\hline
\end{tabular}

Mont: montmorillonite ; K: kaolinite ; I: Illite et Q: quartz

\subsection{Synthèse de MCM-41 à partir de la bentonite Algérienne}

La bentonite est utilisée telle quelle. Pour la synthèse de matériaux mésoporeux, nous avons utilisé la fusion alcaline décrite par Kumar et coll. [8] en remplaçant les cendres volantes de charbon (coal fly ash) par la bentonite. L'approche adoptée, est la dissolution des espèces de silicium et d'aluminium qui existent dans la bentonite en employant la méthode de fusion. La procédure de fusion commence en mélangeant la bentonite en poudre avec l'hydroxyde de sodium pour un rapport en poids, bentonite / $\mathrm{NaOH}$ de 1: 1.2. L'ensemble est chauffé à $550^{\circ} \mathrm{C}$ pendant $1 \mathrm{~h}$ sous l'air. La masse fondue obtenue est refroidie à la température ambiante pendant une nuit. Après cette étape, la bentonite fondue est mélangée avec de l'eau déminéralisée pour un rapport en poids de 1:4 (bentonite/eau). Le mélange est vieilli pendant 1 jour à la température ambiante sous agitation. La suspension résultante est séparée par centrifugation pour obtenir le surnageant. Pour la synthèse d'un échantillon de MCM-41, on mélange $0.867 \mathrm{~g}$ de CTMABr, $15 \mathrm{~mL}$ d'eau et $0.75 \mathrm{~g}$ de $\mathrm{NH}_{4} \mathrm{OH}$ avec $40 \mathrm{~mL}$ du surnageant sous agitation pendant $1 \mathrm{~h}$. Le pH de l'hydrogel est ajusté à des valeurs différentes en utilisant l'acide acétique dilué $(1 \mathrm{M})$. Pour le traitement hydrothermal, le mélange obtenu est porté à différentes températures. Les produits ainsi obtenus sont filtrés et lavés plusieurs fois avec de l'eau déminéralisée puis séchés dans l'étuve à $100{ }^{\circ} \mathrm{C}$ pendant 24 heures. Les échantillons sont 
calcinés sous air à $550{ }^{\circ} \mathrm{C}$ pour une durée de 12 heures.

\subsection{Caractérisation}

Les diffractogrammes de poudre ont été enregistrés sur un diffractomètre de type Bruker AXS équipé d'une anticathode en cobalt $(\lambda=1.788970 \AA$ Å). Adsorption-désorption d'azote à $77 \mathrm{~K}$ est réalisé sur un appareil Microméritics GEMINE 2375, les échantillons sont dégazés à $200{ }^{\circ} \mathrm{C}$ pendant 24 heures sous vide poussé. La surface spécifique $\left(\mathrm{S}_{\mathrm{BET}}\right)$ est calculée par la méthode BET [29], à partir des données de pression relative entre 0.04 à 0.2 en $\mathrm{P} / \mathrm{P}_{0}$. Les observations de microscopie électronique en transmission ont été réalisées sur un microscope Philips CM20 lié à un EDXS (energy dispersive analysis by X-ray spectroscopy).

\section{CONCLUSION}

Dans cette étude, nous avons démontré que la bentonite de Maghnia, peut être utilisée comme une source d'aluminosilicate pour la synthèse de Al-MCM-41. En diminuant la température de synthèse, il est nécessaire de diminuer la valeur du $\mathrm{pH}$ de synthèse pour former la phase hexagonale la mieux ordonnée et éliminer la phase lamellaire. Une incorporation importante d'aluminium fait diminuer la surface spécifique, la valeur la plus élevée est trouvée pour l'échantillon préparé à $100{ }^{\circ} \mathrm{C}$ et $\mathrm{pH}=9.5$. Les rapports $\mathrm{Si} / \mathrm{Al}$ des matériaux mésoporeux synthétisés sont plus élevés que le Si/Al dans la bentonite naturelle (3.2) et proche de celui de la bentonite fondue (8.07). Apparemment, le choix de la température et le rapport bentonite $/ \mathrm{NaOH}$ pour la fusion alcaline ne nous a pas permis d'extraire l'aluminium complètement. L'incorporation élevée d'aluminium est la cause des faibles surfaces spécifiques des échantillons. Par analyse EDX, nous avons confirmé qu' aucune impureté telle que $\mathrm{Fe}, \mathrm{Ca}$, ou K, n'est détectée donc le matériau obtenu est purement aluminosilicaté.

\section{REMERCIEMENTS}

L'auteur (T. Ali-dahmane) tient à remercier tous les membres du Laboratoire Environnement et Minéralurgie (LEM. Nancy, France) ou j'ai pu bénéficier d'un environnement scientifique et technique de qualité pour réaliser ce travail. 


\section{REFERENCES}

[1] Cestors Yand Haller G. L, Microporous and Mesoporous Materials, 2001, 43, 171, doi: S 1387-1811 (00) 00325-5

[2] Shylesh S and Singh A.P, Journal catalysis, 2005, 233, 359, doi: 10.1016/j.jcat.2005.05.001

[3] Gallo R, Pastore O, Schuchardt U, Journal of Non-Crystalline Solids, 2008, 354 (15-16), doi: 10.1016/j.jnoncrysol.2007.10.010

[4] Fontes M. S. B, Melo D. M. A, Costa C. C, Braga R. M, Melo M. A. F, Alves J. A.B and Silva M. L. P, Ceramica, 62, 2016, 85, doi: 10.1590/0366-69132016623611966

[5] Ali-Dahmane T, Brahmi L, Hamacha R and Benguddach R, Ann. Chim. Sci. Mat., 2017, 40 (3-4), 149, doi: 10.3166/acsm.40.149-163

[6] Singer A, Bergaut V. Environ. Sci. Technol., 1995, 29, 1748, doi: 10.1021/es00007a009

[7] Chang H. L, Chun C. M, Aksay I. A and Shih W. H, Ind. Eng. Chem. Res., 1999, 38, 973, doi: $10.1021 /$ ie $980275 b$

[8] Kumar P, Oumi N, Yamana Y and Sano K, Journal of Materials Chemistry, 2001, 11, 3285, doi: 10.1039/b005769j

[9] Liu H, Bao X, Wei W, Kong J and Shi G, Microporous and Mesoporous Materials, 2003, 478, 59, doi: 10.1016/j.micromeso.2003.09.002

[10] Kang F, Wang Q and Xiang S, Materials Letters, 2005, 59, 1426, doi: 10.1016/j.matlet.2005.05.032

[11] Miao S, Liu Z, Ma H, Han B, Du J, Sun Z and Miao Z, Microporous and Mesoporous Materials, 2005, 83, 277, doi: 10.1016/j.micromeso.2005.05.006

[12] Sanhueza V, Lopez-Escobar L, Kelm U and Ruby C, J. Chem. Technol. Biotechnol, 2006, 81, 614, doi: 10.1002/jctb.1443

[13] Wu X. W, Li J. H, Zhang J and Li Z. H, Journal of Colloid and Interface Science, 2007, 315, 555, doi: 10.1016/j.jcis.2007.06.074

[14] Adjdir M, Ali-Dahmane T, Weidler P. G, Friedrich F, Scherer T, Applied Clay Science, 2009, 46, 185, doi: 10.1016/j.clay.2008.11.009

[15] Yang H, Deng Y, Du C and Jin S, Applied Clay Science, 2010, 47, 351, doi: 
10.1016/j.clay.2009.12.021

[16] Majchrzak-Kuçeba I, J. Therm. Calorim., 2012, 107, 911, doi: $10.1007 / \mathrm{s} 10973-011-1908-8$

[17] Schwanke J, Lopes C. W and Castella Pergher S. B, Materials Sciences and applications, $2013,4,68$.

[18] Ghorbani F, Younesi H, Mehraban Z, Sabri celik M, Ghoreyshi A. and Anbia M, J of Taiwan Institute of chemical Engineers, 2013, 44, 821.

[19] Ali-Dahmane T, adjdir M, Hamacha R, Villieras F, Bengueddach A and, Weidler P, C. R. Chimie, 2014, 17, 1, doi: 10.1016/j.crci.2012.12.017

[20] Wang A, Kabe T, Chemistry Communication, 1999, 20, 2067, doi: 10.1039/a906275k

[21] Shigemoto N, Hayashi H and Miyaura K, Journal of Materials Science, 1993,28, 4781, doi: 10.1007/BF00414272

[22] Luechinger M, Frunt L, Pirngruber G. D and Prins R, Microporous Mesoporous Mater, 2003, 64, 203, doi: 10.1016/S1387-1811(03)00483-9

[23] Busio M, Janchen J, van Hooff J. H. C, Microporous Materials, 1995, 5, 211, doi: 10.1016/0927-6513(95)00063-1

[24] Hui K.S, Chao C. Y .H, Journal of Hazardous Materials, 2006, B137, 1135, doi: 10.1016/j.jhazmat.2006.03.050

[25] Sing K. S. W, Everett D. H, Haul R. A. W, Moscou L, Pierotti R. A, Rouquerol J and Siemieniewska T, Pure Appl. Chem., 1985, 57, 603, doi: 10.1351/pac198557040603

[26] Adjdir M, Ali-Dahmane T and Weidler P, C. R. Chimie, 2009, 12, 793, doi: 10.1016/j.crci.2008.09.014

[27] Ryoo R and Kim J. M, J. Chem. Soc. Chem. Commun, 1995, 7, 711, doi: $10.1039 / \mathrm{C} 39950000711$

[28] Edler K. J and White J. W, Chem. Mater, 1997, 9 (5), 1226, doi: 10.1021/cm9606033

[29] Brunauer S, Emmett P. H and Teller E, J. Am. Chem. Soc., 60 (1938) 309, doi: $10.1021 / \mathrm{ja} 01269 \mathrm{a} 023$.

\section{How to cite this article:}

Ali-Dahmane T, Brahmi L, Hamacha R, Villieras F and Bengueddach A. Synthesis of MCM-41 nanomaterial from Algerian bentonite: Influence of synthesis $\mathrm{pH}$. J. Fundam. Appl. Sci., 2016, 9(2), 636-649. 